\title{
Cancer Versus COVID-19: A Coordinated Disease Outbreak Response System (DORS) to Combat COVID-19 at the National Cancer Centre Singapore
}

\author{
Ravindran Kanesvaran, ${ }^{1,2}{ }_{M D}$, MRCP (UK), FAMS (Med Onco), Claramae S Chia, ${ }^{2,3}$ MBBS, MMed (Surg), FRCS (Edin),

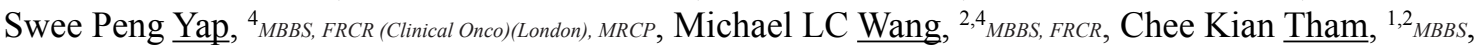 \\ Soon Thye Lim, ${ }^{1,2}$ MBBS, MRCP (UK), William YK Hwang, ${ }^{2,5}$ MBBS, FRCP (London), FAMS, Jin Wei Kwek,${ }^{2,6}{ }_{M B B S}$, FRCR (UK), FAMS
}

It is now known that cancer patients are at higher risks of COVID-19 and its associated complications. Early data from Wuhan showed that cancer patients are at increased risk of mortality compared to non-cancer patients afflicted with COVID-19. ${ }^{1,2}$ In the data, the mortality rate was nearly $29 \% .^{1}$ Another study done in the US showed a similar finding of increased mortality among patients with cancer who were younger than 50 years of age. ${ }^{3}$

The National Cancer Centre Singapore (NCCS) is the largest comprehensive academic cancer centre in Southeast Asia with 155,422 outpatient consultations in 2019. With the case load that it is handling, a coordinated effort is required at the centre to ensure business continuity while maintaining patient and staff safety. With this pandemic overwhelming many healthcare systems globally, we aim to share our experience of our coordinated Disease Outbreak Response System (DORS) team in response to COVID-19.

Upon the diagnosis of the first confirmed COVID-19 case in Singapore on 23 January 2020, ${ }^{4}$ a DORS taskforce was mobilised immediately within NCCS. We have been prepared for this since the severe acute respiratory syndrome (SARS) (caused by SARS-CoV) pandemic hit Singapore in 2003, ${ }^{5}$ with annual DORS exercises conducted within the centre, as well as at the Singapore General Hospital (SGH) campus (on which NCCS is situated). Infection control protocols were established by the DORS taskforce, which included regular personal protective equipment (PPE) training and DORS protocol refresher sessions for our NCCS staff. This time, the NCCS DORS taskforce coordinated the development and implementation of COVID-19 policies and protocols in conjunction with the other hospitals and specialty centres (which together make up the cluster of healthcare institutions known as
Singapore Health Services). Important policy considerations included protection of our staff and immunocompromised cancer patients from COVID-19, manpower preservation, prevention of burnout due to manpower shortage, prudent use of our resources for oncological care with infection control, and the implementation of safe distancing measures. The DORS taskforce was mobilised rapidly in response to the COVID-19 outbreak, and ensured a smooth transition from DORSCON (Disease Outbreak Response System Condition) Yellow to Orange on 7 February 2020. ${ }^{5}$ The DORSCON system is a colour-coded framework developed by the Ministry of Health of Singapore, to represent the severity and spread of an outbreak and the measures taken to address this. There are 4 statuses: Green (when it is a mild outbreak), Yellow, Orange and Red (which signifies a severe disease outbreak).

\section{Patient and Staff Safety Paramount}

The first step was to ensure strict screening and triage measures at the entrance of NCCS. Thermal scanning and questionnaire-based screening were carried out on all patients and visitors at this checkpoint. Only patients with no fever, acute respiratory symptoms or relevant travel history or contact history with COVID-19 patients or clusters were allowed into the main NCCS building. A febrile patient, or one meeting the Ministry of Health $(\mathrm{MOH})$ criteria for a suspect case, would be escorted to a fever screening clinic for subsequent management. The setting up of the NCCS fever clinic was initiated since DORSCON Orange began. This is in keeping with international guidelines, which recommended an appropriate assessment area for patients at risk of neutropaenia getting COVID-19. ${ }^{6}$ Patients who have fever or respiratory symptoms from their primary tumours or treatment-related symptoms are segregated from those suspected of having

\footnotetext{
${ }^{1}$ Division of Medical Oncology, National Cancer Centre Singapore, Singapore

${ }^{2}$ Oncology Academic Programme, Duke-NUS Medical School, Singapore

${ }^{3}$ Division of Surgery and Surgical Oncology, National Cancer Centre Singapore, Singapore

${ }^{4}$ Division of Radiation Oncology, National Cancer Centre Singapore, Singapore

${ }^{5}$ Department of Haematology, National Cancer Centre Singapore, Singapore

${ }^{6}$ Division of Oncological Imaging, National Cancer Centre Singapore, Singapore

Address for Correspondence: Dr Kwek Jin Wei, Division of Oncologic Imaging, National Cancer Centre Singapore, 11 Hospital Crescent, Singapore 169610.

Email: kwek.jin.wei@singhealth.com.sg
} 
COVID-19, so that the former can be allowed to proceed with their treatment. Suspect cases were sent to the SGH Department of Emergency Medicine to be swabbed for SARS-CoV-2 polymerase chain reaction (PCR) testing. Suspected high-risk COVID-19 cases would be admitted to be managed in a designated ward in SGH. This clinic started to send swab samples for SARS-CoV-2 PCR testing as community spread increased. Later, $\mathrm{MOH}$ recommended that cancer patients on chemotherapy presenting with acute respiratory infection of any duration should be swabbed and tested too. This 'swab and go' effort is ongoing at the time of this manuscript's preparation. To ensure safe social distancing and avoid overcrowding at the centre, we limited accompanying visitors to one per patient. Since the beginning of the circuit breaker period (the Singapore definition of a lockdown), the rule was tightened to disallow any accompanying visitors for patients undergoing chemotherapy in the ambulatory treatment unit. For patients requiring assistance with mobility, a team of ushers was mobilised to assist. All outpatients, visitors and vendors cleared to enter NCCS were required to wear a mask. Non-medical staff who did not need to work on-site telecommuted where possible.

All medical, nursing and allied healthcare personnel were segregated into teams to ensure business continuity. Medical oncology teams were further geographically segregated into an outpatient team, and teams for specific wards in SGH, to minimise cross-contamination and loss of workforce due to quarantine in case of staff infection. Audits of new and planned cases were done remotely via teleconferencing. Cancer surgeries were allowed to proceed as planned, but all non-cancer surgeries were postponed where possible. The reduction of patient volume was necessary to allow safe distancing measures as well as sustainability of a segregated team model. Outpatient appointments for patients on cancer surveillance were deferred. Teleconsult was developed to reduce patient visits to the centre. To decrease patient dwell time and overcrowding in NCCS, online electronic payment was encouraged. Home delivery of non-urgent and non-controlled medications was carried out as well.

\section{Impact on Patient Care}

The slew of measures rolled out above did have a positive impact in curtailing the spread of COVID-19 infections among our patients. To date, only 3 NCCS cancer patients have been reported to have contracted COVID-19. More importantly, none of them contracted it from a visit to NCCS as they were all infected in the community. Equally important was the fact that no NCCS staff has been infected with COVID-19. However, the pandemic and the measures instituted above have had a major impact on the number of patient-visits to the centre, including the number of new cases diagnosed (Table 1).

There was a drop in the new diagnoses of cancer presented to NCCS by nearly $26 \%$ compared to the same duration in 2019. This was consistent with international data. ${ }^{7}$ We postulate a few reasons for this occurrence here in Singapore. Firstly, there was limited community cancer screening on-going owing the pandemic. Patients were also more fearful of presenting to a medical facility or even see their general practitioners (GPs) due to the fear of contracting the virus, and hence were tolerating their symptoms. In the Netherlands, 2 other issues were highlighted as potential causes for the drop in patient attendance. One was the diversion of resources in the hospitals to support COVID-19 cases, leading to a delay in getting appointments to diagnose cancer. Another reason was the use of telemedicine to consult GPs during this period, which may have led to a delay in getting certain patients investigated and diagnosed for cancer because of the difficulty in identifying critical signs and symptoms. ${ }^{7}$ The fewer new cancer diagnoses now may potentially lead to a rapid rise in cases in the post COVID-19 period, as these cases will be diagnosed later. This will impact NCCS's workload and may overwhelm our ability to treat these patients in a timely manner. We may also see more late-stage cancer at presentation. In terms of follow-up cases, there was a deliberate rescheduling of stable follow-up cases to a later date in order to reduce congestion in the centre. This led to about a $30 \%$ drop in our follow-up patient-load.

Clinical trial enrolment was affected during this period too. No new trial enrolment was allowed during the circuit breaker period. However, existing patients on potentially life-saving clinical trials were allowed to continue. This led to an overall decrease in patients enrolled in clinical trials, and was consistent with data from the US. ${ }^{8}$ However, it must be noted that, despite the postponement of follow-up appointments for stable patients, there was no drop in the active treatment instituted in patients (as reflected by chemotherapy chair utilisation, radiotherapy visits and cancer related surgery) over the same period one year ago.

\section{Silver Lining: The Future of Oncology Redefined}

While the pandemic has taken its toll on the lives of cancer patients worldwide, with other indirect negative outcomes on survival and morbidity on our local cancer patients, there is a silver lining to this. A number of measures we had instituted to combat this scourge has changed the quality of cancer care for our patients for the better. The pharmacy drug courier service has been 
Table 1. Data collected during DORSCON Orange at NCCS from February to May/July 2020 compared to same period in 2019

\begin{tabular}{|lll|}
\hline & Total Number (February-July 2019) & $\begin{array}{l}\text { Total Number (February-July 2020) } \\
\text { (\% Difference) }\end{array}$ \\
\hline Outpatient clinic load & & \\
\hline Outpatient clinic (total number of patients) & 58,562 & $43,943(-24.9 \%)$ \\
\hline Number of first-visit consultations & 6,937 & $4,852(-24.2 \%)$ \\
\hline Number of follow-up consultations & 52,165 & $39,091(-25.1 \%)$ \\
\hline Chemotherapy/treatment chair utilisation & 19,047 & $16,972(-10.9 \%)$ \\
\hline Radiation treatment visits & 17,915 (February-May 2019) & 15,914 (February-May 2020) (-7.4\%) \\
\hline Cancer-related surgery & 670 (February-May 2019) & 672 (February-May 2020) (+0.3\%) \\
\hline COV & - & 234 \\
\hline Number of COVID-19 PCR swabs taken & - & 3 \\
\hline Number of confirmed COVID-19 cases amongst NCCS patients & - & 0 \\
\hline Number of confirmed COVID-19 cases amongst NCCS staff & - & $24(-58.6 \%)($ Feb-May 2020) \\
\hline Research & & 58 (February-May 2019) \\
\hline Number of patients recruited into clinical trials (total)
\end{tabular}

DORSCON: Disease Outbreak Response System Condition; NCCS: National Cancer Centre Singapore; PCR: polymerase chain reaction

ramped up, with about half of NCCS prescriptions being sent to patients' homes. Telemedicine is another key initiative that will help reduce congestion in the centre and provide a convenient option for all our patients. Restriction on the number of patient consults (in order to enforce better safe distancing) per clinic resource, has also shortened wait times and crowding outside the clinic. Patient experience has also improved, with reports of longer, more meaningful consultations with their doctors. Recently, a new committee has been formed by NCCS to consolidate some of the positive changes described above, and to create new patient-centric policies that will not only ensure safe distancing in this COVID-19 era, but also ensure a better patient experience when visiting NCCS in time to come. COVID-19 has been the most difficult challenge NCCS has faced in its 20-year history, but as with all challenges, it presented an opportunity to not only keep our patients safer, but also provided a golden opportunity to change the face of cancer care in Singapore for the better.

\section{REFERENCES}

1. Zhang L, Zhu F, Xie L, et al. Clinical characteristics of COVID-19infected cancer patients: a retrospective case study in three hospitals within Wuhan, China. Ann Oncol 2020;31:894-901

2. Dai M, Liu D, Liu M, et al. Patients with cancer appear more vulnerable to SARS-CoV-2: a multicenter study during the COVID-19 outbreak. Cancer Discov 2020;10:783-91.

3. Miyashita H, Mikami T, Chopra N, et al. Do patients with cancer have a poorer prognosis of COVID-19? An experience in New York City. Ann Oncol 2020;31:1088-9.

4. Hsu LY, Chia PY, Lim JF. The novel coronavirus (SARS-CoV-2) epidemic. Ann Acad Med Singap 2020;49:105-7.

5. Goh KT, Cutter J, Heng BH, et al. Epidemiology and control of SARS in Singapore. Ann Acad Med Singap 2006;35:301-16.

6. Burki TK. Cancer guidelines during the COVID-19 pandemic. Lancet Oncol 2020;21:629-30.

7. Dinmohamed AG, Visser O, Verhoeven RHA, et al. Fewer cancer diagnoses during the COVID-19 epidemic in the Netherlands. Lancet Oncol 2020;21:750-1.

8. Unger JM, Blanke CD, LeBlanc M, et al. Association of the coronavirus disease 2019 (COVID-19) outbreak with enrollment in cancer clinical trials. JAMA Netw Open 2020;3:e2010651. 